\title{
Achievement of ultralow emittance coupling in the Australian Synchrotron storage ring
}

\author{
R. Dowd, M. Boland, G. LeBlanc, and Y-R. E. Tan \\ Australian Synchrotron, 800 Blackburn Road, Clayton, 3168, Australia
}

(Received 16 April 2010; published 29 January 2011)

\begin{abstract}
Investigations into producing an electron beam with ultralow vertical emittance have been conducted using the Australian Synchrotron $3 \mathrm{GeV}$ storage ring. A method of tuning the emittance coupling $\left(\epsilon_{y} / \epsilon_{x}\right)$ has been developed using a machine model calibrated through the linear optics from closed orbits method. Direct measurements of the beam emittance have not been possible due to diagnostic limitations, however two independent indirect measurements both indicate a vertical emittance of $1.2-1.3 \mathrm{pm} \mathrm{rad}\left(\epsilon_{y} / \epsilon_{x}=0.01 \%\right)$. Other indirect measurements support the validity of these results. This result is the smallest vertical emittance currently achieved in a storage ring.
\end{abstract}

DOI: $10.1103 /$ PhysRevSTAB.14.012804

PACS numbers: 29.27.Fh, 41.75.Ht

\section{INTRODUCTION}

The Australian Synchrotron is a newly operating 3rd generation light source facility located in Melbourne, Australia. The $3 \mathrm{GeV}$ storage ring is 216 meters in circumference and can store a beam of up to $200 \mathrm{~mA}$ current. A storage ring design overview can be found in [1].

The storage ring is a modified Chasman-Green-type lattice, designed for a horizontal emittance of $14 \mathrm{~nm} \mathrm{rad}$ with dispersion free straights. For user operations a horizontal emittance of $10.4 \mathrm{~nm}$ rad is achieved by allowing a $0.1 \mathrm{~m}$ dispersion in the straights. The $0.1 \mathrm{~m}$ dispersion setting, while not the minimal horizontal emittance, achieves the minimal horizontal beam size when taking into account both emittance and dispersion contributions. The storage ring is divided into 14 identical sectors, each containing a $10.9 \mathrm{~m}$ arc and $4.5 \mathrm{~m}$ straight section. Of the 14 straights, two are used for rf cavities, one for diagnostics and one for beam injection. Each arc segment contains two dipole magnets (with defocusing gradients), six quadrupole, and seven sextupole magnets, shown in Fig. 1. Orbit and coupling corrections are achieved via additional windings on the sextupole magnets, fed by power supplies independent of the main magnet supply. Four sextupole magnets in each arc have the required windings to act as skew quadrupoles, although only two of these are currently powered, giving a total of 28 skew quadrupole magnets around the storage ring. The power supplies for these skew quadrupoles are bipolar and can supply a current of up to $5 \mathrm{~A}$, which delivers a maximum $k$ strength of 0.02 per skew quadrupole.

\section{BEAM DIAGNOSTICS}

There are two diagnostic beam lines at the Australian Synchrotron, an $\mathrm{x}$ ray and an optical beam line. Details of the diagnostics beam lines can be found in Ref. [2].

The x-ray diagnostic beam line (XDB) uses the x-ray light from a bending magnet and passes it through a pinhole array. The multiple pinhole images formed are then projected onto a fluorescent screen and imaged by a CCD camera. By measuring the beam size and knowing the beta functions and dispersion at the source point, it is possible to extract the beam emittance from this image. The CCD camera and beam line optics allow for beam size measurements with $5 \mu \mathrm{m}$ resolution. However, the resolution limit from diffractive effects is $60 \mu \mathrm{m}$ [3], which limits the resolution of vertical emittance measurements on this beam line to values above $10 \mathrm{pm}$. Additional effects due to fast beam movement during relatively slow CCD integration and uncertainties in the point spread function of the system make the beam line currently unsuitable for precision beam size measurements.

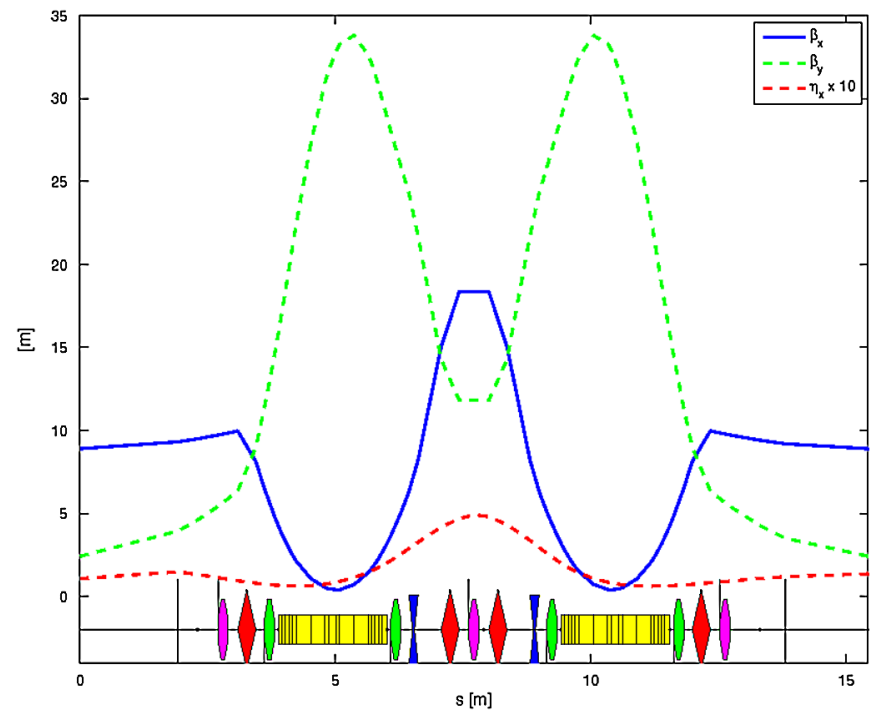

FIG. 1. Storage ring magnets and lattice functions. Dipoles (yellow), quadrupoles (red/blue), and sextupoles (green/purple) are shown. Skew quadrupoles are located in the 2nd and 6th sextupole. Betatron tunes are $\nu_{x}=13.29, \nu_{y}=5.216$. 

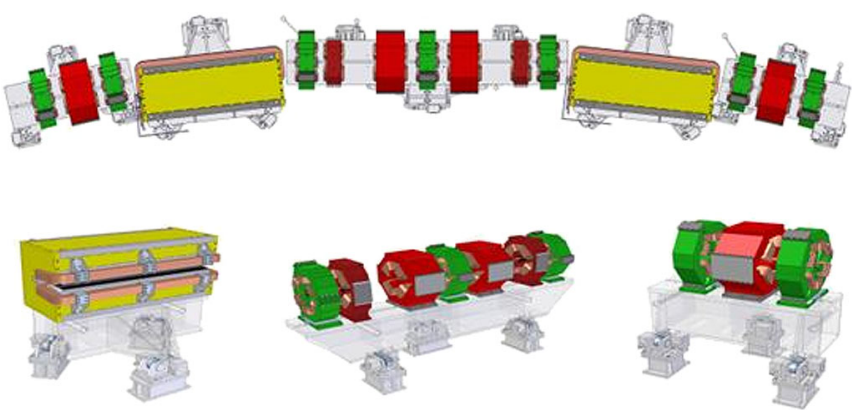

FIG. 2. Layout of magnets and support girders showing adjustment points. The top image shows a top view of the arc sector magnets. The bottom image illustrates the three types of support girder and adjustable supports used in the storage ring.

\section{ALIGNMENT}

\section{A. Magnet alignment}

The storage ring multipole magnets in each arc sector are mounted onto one of three adjustable girders, with the dipole being independently supported and aligned, and are shown in Fig. 2. The girder support system and alignment techniques are detailed in [4].

In the middle of 2008 a full storage ring survey and realignment was conducted. The individual magnet vertical offsets before and after this realignment are shown in Table I. After the realignment, the uncorrected emittance ratio in the storage ring was found to have decreased from $0.1 \%$ to $0.06 \%$, as measured by the technique described in Sec. IV.

\section{B. Beam position monitor (BPM) resolution and beam based alignment}

The Australian Synchrotron storage ring contains a total of 98 beam position monitors for orbit monitoring and control. I-tech "Libera Electron" units are used as the BPM electronics, allowing high precision measurements and full integration into the experimental physics and industrial control system (EPICS) control environment [5]. Currently, the orbit position resolution achieved at the Australian Synchrotron is $0.1 \mu \mathrm{m}$ (rms) with nominal fill pattern and current. Beam based alignment is conducted using standard quadrupole shunting methods. Each BPM is calibrated to the magnetic center of the quadrupole nearest

TABLE I. Alignment survey results before and after realignment. The numbers show the standard deviation of the vertical position of all the magnets with respect to a common plane. The error on these numbers indicates the fiducial and assembly errors, which cannot be corrected and thus represent the lowest achievable alignment error.

\begin{tabular}{lccc}
\hline \hline Survey & Sextupole & Quadrupole & Dipole \\
\hline Before 07/2008 & $76 \pm 51 \mu \mathrm{m}$ & $58 \pm 16 \mu \mathrm{m}$ & $45 \pm 6 \mu \mathrm{m}$ \\
After 07/2008 & $55 \pm 51 \mu \mathrm{m}$ & $26 \pm 16 \mu \mathrm{m}$ & $18 \pm 6 \mu \mathrm{m}$ \\
\hline \hline
\end{tabular}

it and a resolution of about $10 \mu \mathrm{m}$ is achieved. This compares favorably with the mechanical alignment resolution of the BPMs, which is less than $20 \mu \mathrm{m}$. Beam based alignment is conducted quite regularly, with a full ring recalibration done at least every two months and after any realignment or BPM recabling. Orbit correction is achieved with 42 horizontal and 56 vertical corrector magnets. Typical rms orbit deviation with $0.25 \mathrm{~Hz}$ global orbit feedback and $10 \mathrm{~Hz}$ BPM acquisition is $<20 \mu \mathrm{m}$ horizontal and $<10 \mu \mathrm{m}$ vertical.

\section{LOCO BASED EMITTANCE ADJUSTMENT}

\section{A. LOCO method}

The method used to control the transverse coupling in our storage ring is an orbit response matrix (ORM) analysis utilizing the linear optics from closed orbits (LOCO) algorithm [6]. LOCO is widely used at synchrotron light sources around the world for analysis and correction of linear optics and is used extensively at the Australian Synchrotron [7]. The LOCO algorithm combines the orbit response matrix and dispersion measurements from the machine and compares them to a model of the storage ring. It then adjusts user defined model variables in order to best match the behavior of the real machine. Many different model variables may be defined to be used in the LOCO fitting, common variables are: BPM gains and couplings, corrector magnet gains and couplings, multipole magnet strengths, and skew quadrupole components.

These variables are adjusted over several iterations in the model until the model behavior matches the measured machine by creating and minimizing a Jacobian error matrix. The minimization algorithm used by LOCO in this analysis is the Levenberg-Marquardt method [8]. We refer to the final adjusted model as the calibrated model, which can now be used in simulations to predict beam parameters and behavior. Because the model has been calibrated to the real behavior of the machine, simulations using it can take into account the magnet skews, tilts, and gains determined by the ORM fitting. Provided that care has been taken to ensure that the LOCO input data and fits are sensible and the model is accurate, the calibrated model is a very powerful predictor of the real behavior of the beam. We must take care, however, in extrapolating model results to extreme conditions such as in the ultralow vertical emittance regime. Model predictions need to be crosschecked as much as possible against more direct beam measurements before they can be trusted.

Figures 3 and 4 show some of the outputs from the ORM fitting. In Fig. 3(a) we have shown the results of several different LOCO fits of the BPM vertical coupling; each fit is denoted by a different color. These fits were done with different coupling settings, ranging from minimal coupling to $17 \%$ (maximum achievable with the skew quadrupoles), yet show a very good consistency of the fitted BPM coupling. Similar results are seen in the horizontal coupling. In 

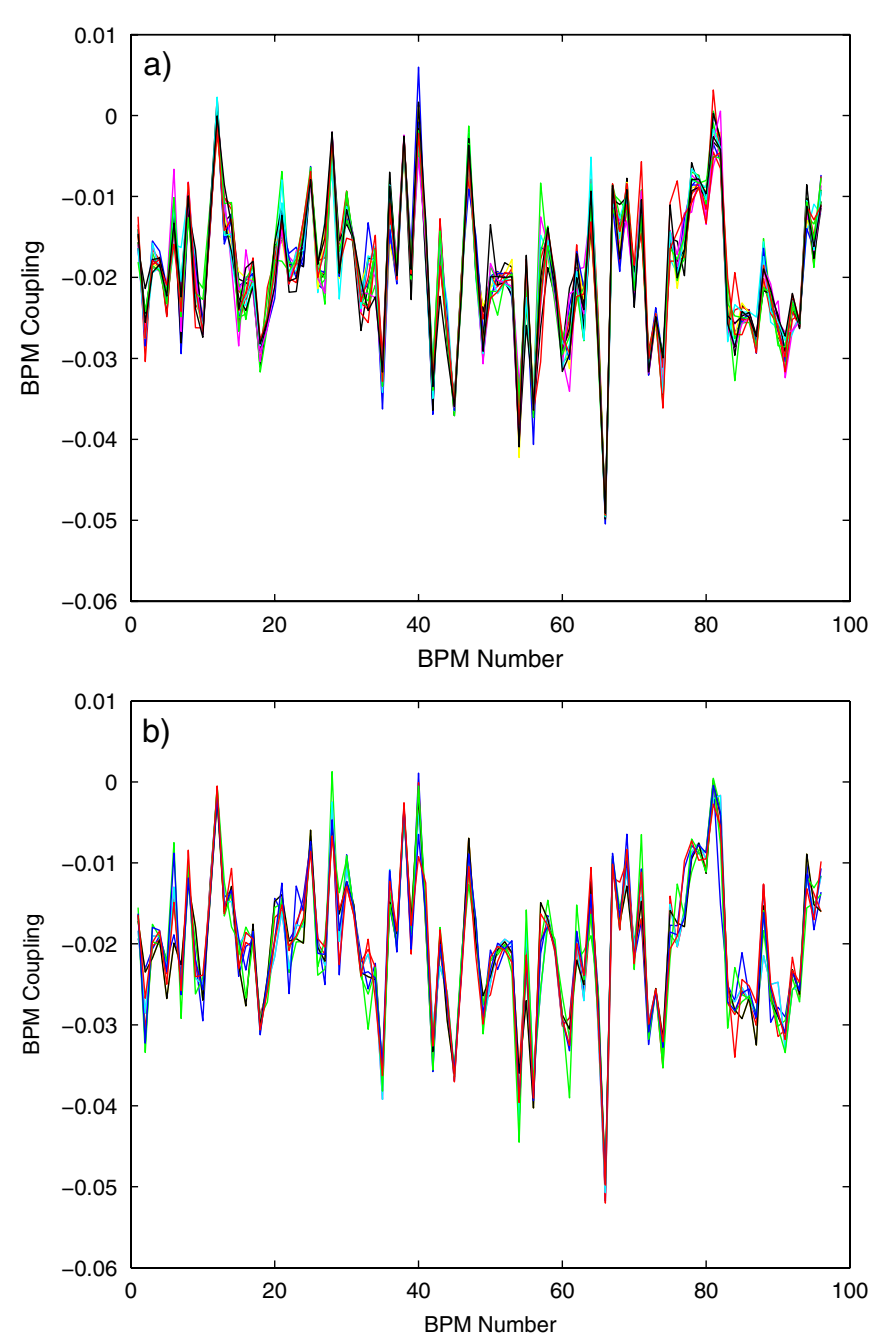

FIG. 3. Plots of LOCO fitted BPM vertical coupling. Plot (a) shows the results over several fits of varying machine condition, Plot (b) shows the results for multiple fits to the same machine configuration.

Fig. 3(b) we compared the BPM vertical coupling results obtained from fitting multiple data sets of the same machine configuration and see the same magnitude spread in the results. Some differences arise in particular BPMs when comparing Figs. 3(a) and 3(b) due to known physical changes in the BPM block or associated electronics between the measurements used to generate these data sets. This comparison demonstrates that error in the ORM fitting process is the most significant cause of difference between the BPM fit parameters for data sets with different machine coupling. This is also seen for the other BPM and corrector magnet parameters, which indicates that changes in machine coupling are correctly being attributed to skew components in the ring magnets and not couplings in the BPMs and correctors. The coupling in Fig. 3 is not distributed around zero due to known electronic cross-talk effects in the BPM front-end electronics and has previously been
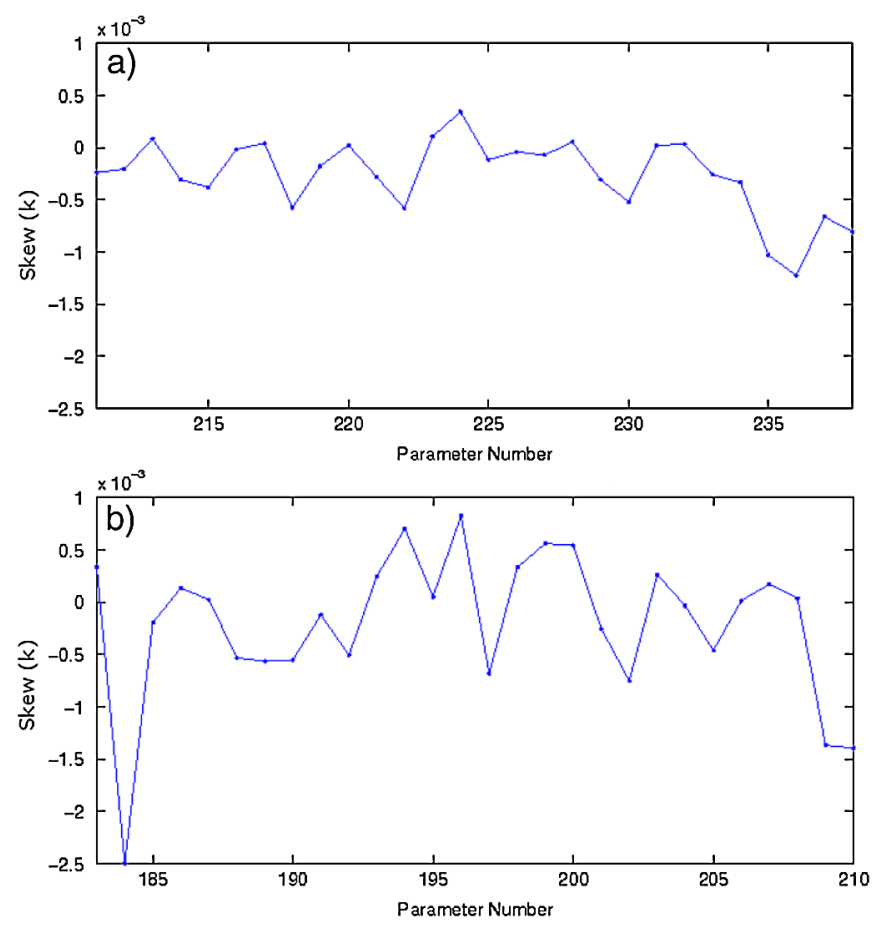

FIG. 4. Examples of LOCO fitted skew quadrupole components for two of the quadrupole families in the storage ring. Plot (a) shows the focusing quadrupoles in the inner, long girder; plot

(b) shows the focusing quadrupoles on the outer, short girders.

shown to have good agreement with calculated couplings based on BPM cable attenuation measurements [9].

\section{B. Girder anomaly}

From Fig. 4 it can be seen that there is a large spike in the fitted skew quadrupole component in the second quadrupole magnet [(plot (b)]. This magnet is on a short girder with two other sextupoles. The sextupoles were also found to have a large skew component assigned to them by the ORM fit. As there are no BPMs between the magnets on this girder, an ORM analysis is not able to distinguish which magnet is the source of the coupling. The fitted skew component is therefore distributed along all three magnets in the fit. The girder was investigated and found to have a significant forward pitch of $600 \mu \mathrm{m}$ over the length of the girder, with the center of the girder believed to be at the correct height. A simulation was performed with a $\pm 300 \mu \mathrm{m}$ vertical misalignment in the sextupoles on either end of the girder. The resulting skew quadrupole components that were produced were of comparable magnitude to those found on the machine by LOCO.

This misalignment had not been seen on the survey data and some investigation was required to reveal the cause. The vertical planing of the girder is done using the tops of the magnets and according to the survey data the magnet tops were all in the correct plane. The origin of the error 
was eventually found when the heights of each magnet were measured and one of the sextupoles was found to be shorter than the others. Removing this magnet from the planing fit looks as if it will restore the girder pitch back to flat. This realignment will be done in the near future.

Finding the cause of this anomaly using data from the LOCO fit supports empirically that the skew data produced by LOCO are reflecting real misalignments on the machine. While this result shows the calibrated model to be accurate for larger misalignments, predictions of the model at smaller coupling settings will need to be verified by other means.

\section{Coupling minimization}

In previous work on coupling control [10], a LOCO model with only skew components at the locations of the skew quadrupole magnets was used. This meant that when LOCO fitted for the coupling in the ring it projected all sources of coupling on these skew quadrupoles. The corresponding skew quadrupole current values would then be inverted and applied back onto the magnets. If the assumption of the model fit was correct, this would effectively cancel out the coupling in the ring. While this method was successful in reducing the coupling considerably and is also used at other storage rings, there were two concerns with this method. One was that LOCO would often fit a skew component to one of the skew quadrupoles that was larger than the ability of the power supply to generate (as was the case for the pitched girder). This meant that we could not apply the correct inverse settings to the machine. The other concern was that in forcing LOCO to only assign a skew component to certain magnets in the ring we were compromising the accuracy of the fit. Both of these issues could result in suboptimal coupling minimization.

To address the above-mentioned concerns for this study, LOCO is made to fit for a skew component in all multipoles. These skew components simulate any possible rolls or misalignments of the multipoles that would give rise to coupling. A LOCO analysis is then performed on the uncorrected lattice (a lattice with all skew quadrupoles turned off) to measure the intrinsic coupling of the ring. The fitted skews are then applied to the lattice model and the horizontal and vertical emittance is calculated using the MATLAB Accelerator Toolbox (AT). This emittance calculation uses particle tracking to determine the equilibrium beam distribution as a function of position around the storage ring following the method in [11]. The horizontal and vertical emittance, local beam tilt, and beam size are all determined. The result of this emittance calculation is then fed to a minimization algorithm which varies the strengths of the skew quadrupole magnets in order to achieve the desired emittance coupling. The minimization algorithm allows for arbitrary emittance ratio settings to be achieved simply by modifying the value that the algorithm is seeking to minimize. It also takes into account the limits
TABLE II. Results from the analysis of the LOCO calibrated models for each emittance ratio setting.

\begin{tabular}{lcc}
\hline \hline Set $\epsilon_{y} / \epsilon_{x}$ & Calculated $\epsilon_{y} / \epsilon_{x}$ & Calculated $\epsilon_{y}(\mathrm{pm})$ \\
\hline $0.0 \%$ & $0.01 \%$ & $1.0 \pm 0.03$ \\
$0.1 \%$ & $0.12 \%$ & $12.2 \pm 0.37$ \\
$0.2 \%$ & $0.23 \%$ & $23.5 \pm 0.71$ \\
$0.3 \%$ & $0.33 \%$ & $33.7 \pm 1.01$ \\
$0.4 \%$ & $0.43 \%$ & $43.9 \pm 1.32$ \\
$0.5 \%$ & $0.54 \%$ & $55.1 \pm 1.65$ \\
$0.6 \%$ & $0.64 \%$ & $65.3 \pm 1.96$ \\
$0.7 \%$ & $0.74 \%$ & $75.5 \pm 2.23$ \\
$0.8 \%$ & $0.84 \%$ & $85.7 \pm 2.57$ \\
$0.9 \%$ & $0.92 \%$ & $93.8 \pm 2.81$ \\
$1.0 \%$ & $1.04 \%$ & $106.1 \pm 3.18$ \\
\hline \hline
\end{tabular}

of the skew quadrupole power supplies, allowing the algorithm to potentially distribute a coupling correction globally in the case where a full local correction is outside the range of a power supply and therefore not achievable. A series of coupling configurations were determined using this procedure, allowing the storage ring coupling to be set in $0.1 \%$ increments from $0.1 \%$ to $1.0 \%$.

These configurations were applied onto the machine and a response matrix and dispersion measurement were taken at each setting. The measurements were then fitted to an uncalibrated model (with no a priori information about the current machine configuration) using LOCO. We now have a LOCO calibrated model for each coupling setting and these are used to determine the emittance coupling and $\epsilon_{y}$. The results derived from these calibrated models are shown in Table II. Errors on the model predictions were estimated by taking ten response matrix measurements at the same machine settings and finding the variance of the calculated emittance ratio. This was done at minimum coupling and $1 \%$ coupling and the proportional error was found to be $2.6 \%$ and $0.5 \%$, respectively. From these results we chose the worst case result and applied a $3 \%$ error to all model predictions.

\section{DISPERSION MINIMIZATION}

The vertical emittance arises from two contributions, emittance from betatron coupling and from vertical dispersion. In the current lattice configuration there is some horizontal dispersion present at the locations of the skew quadrupoles, allowing for the possibility of coupling some of this dispersion into the vertical plane to cancel out vertical dispersion.

The minimization algorithm used above does not distinguish between the two contributions when seeking to minimize the total emittance coupling, $\epsilon_{y} / \epsilon_{x}$. Since the betatron coupling and vertical dispersion can both be affected by the same skew quadrupoles, it is not necessarily 




FIG. 5. Result of a typical dispersion measurement. Effects of BPM coupling can clearly be seen as the peaks of the horizontal dispersion (top) appear in the vertical dispersion plot (bottom).
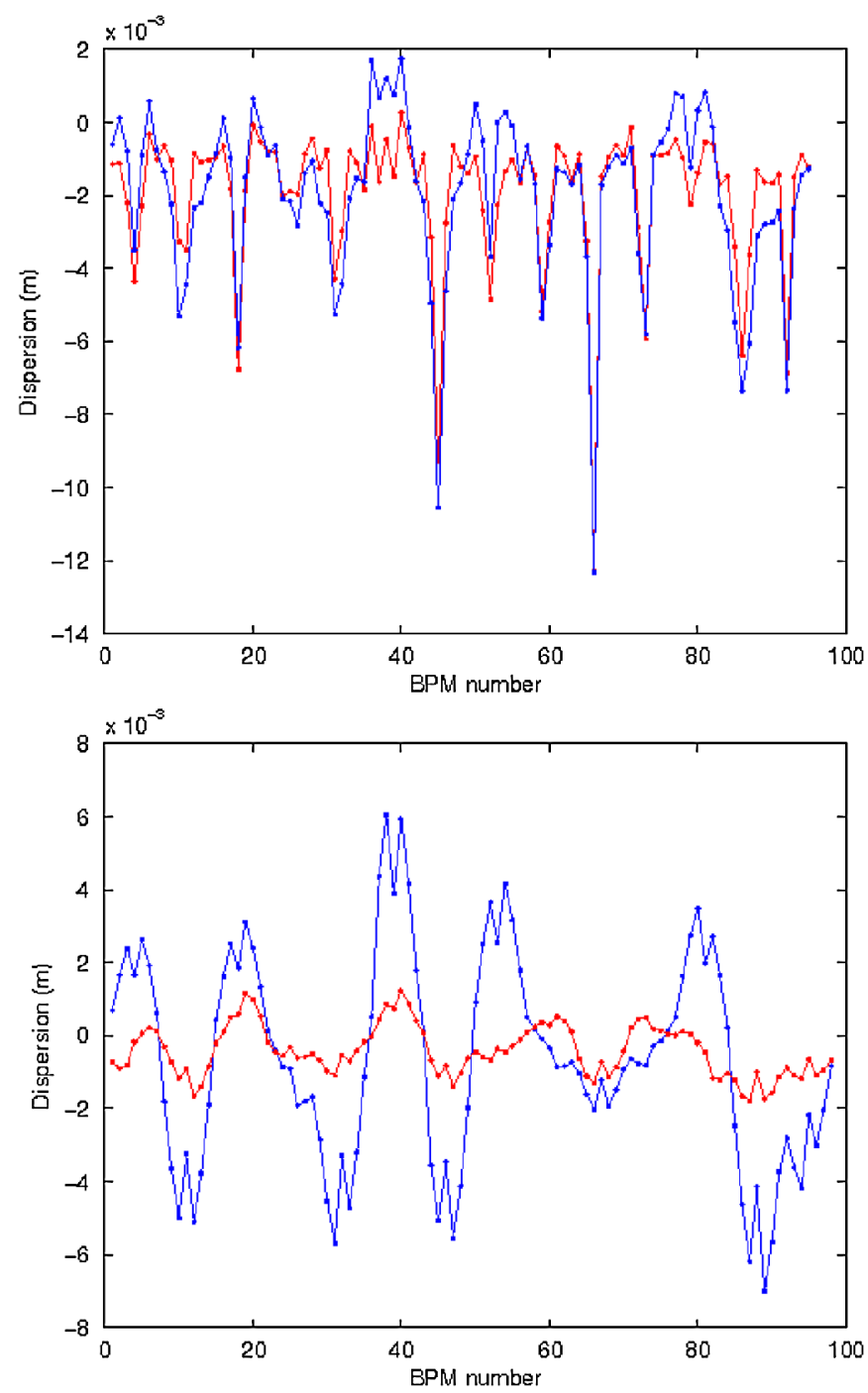

FIG. 6. Dispersion measurement of uncorrected lattice (blue) and dispersion minimized lattice (red). The top plot shows the raw dispersion data, while the bottom plot has the BPM coupling effect removed. Both show a clear reduction in vertical dispersion. possible to minimize both quantities simultaneously. In the total emittance coupling minimization, the rms vertical dispersion was decreased from $3.4 \pm 0.1 \mathrm{~mm}$ to $2.2 \pm 0.1 \mathrm{~mm}$ (compared to a model predicted result of $2.3 \mathrm{~mm}$ ). It is believed that lowering it further using the skew quadrupoles will introduce more betatron coupling and thus raise the overall vertical emittance.

Unlike the emittance, the vertical dispersion can be measured directly with our BPMs. We decided to use this as a check of the minimization procedure by modifying the algorithm such that it minimized only the vertical dispersion. A direct measurement could then be made of the vertical dispersion and compared to the value expected from the LOCO calibrated model.

One complication in this measurement is the BPM coupling, which causes horizontal dispersion to also be picked up on the vertical dispersion measurement. This is most obvious as a series of peaks in the vertical dispersion that correspond to the center of the dipole magnets (the largest point of horizontal dispersion). This effect is illustrated in Fig. 5. To remove this, we can use the LOCO calibrated model, and plot the dispersion with the BPM coupling set to zero. Since we have previously verified that the BPM couplings found by LOCO are consistent between data sets, this new dispersion measurement is still a direct measurement, just with a common factor subtracted. An error estimate of this process was obtained by again analyzing a series of ten measurements and finding the variance of resulting dispersion. An error of $4 \%$ was found.

When the dispersion minimization is performed, the model predicts a final $\mathrm{rms}$ dispersion of $0.946 \mathrm{~mm}$, with an emittance coupling of $1.0 \%$. These settings were applied to the machine and a response matrix and dispersion measurement taken and fed into LOCO. The LOCO fitting found the machine now had a rms vertical dispersion of $0.907 \pm 0.04 \mathrm{~mm}$ with an emittance coupling of $0.88 \pm$ $0.3 \%$. The comparison of the dispersion measurements is shown in Fig. 6.

\section{TUNE CROSSING MEASUREMENTS}

A tune crossing measurement gives an estimate of the amount of betatron coupling present. While this measurement will not give the full emittance coupling present, the betatron coupling determined from it should be able to be combined with the vertical dispersion measurement to give an estimate of the vertical emittance. This result can then be compared with the values predicted by the calibrated model.

The betatron coupling ratio, $g$, is given by [12]

$$
g=\frac{(|C| / \Delta)^{2}}{(|C| / \Delta)^{2}+2}
$$

Where $\Delta$ is the nominal distance of the tunes from the difference resonance, $|C|$ is the modulus of the coupling 

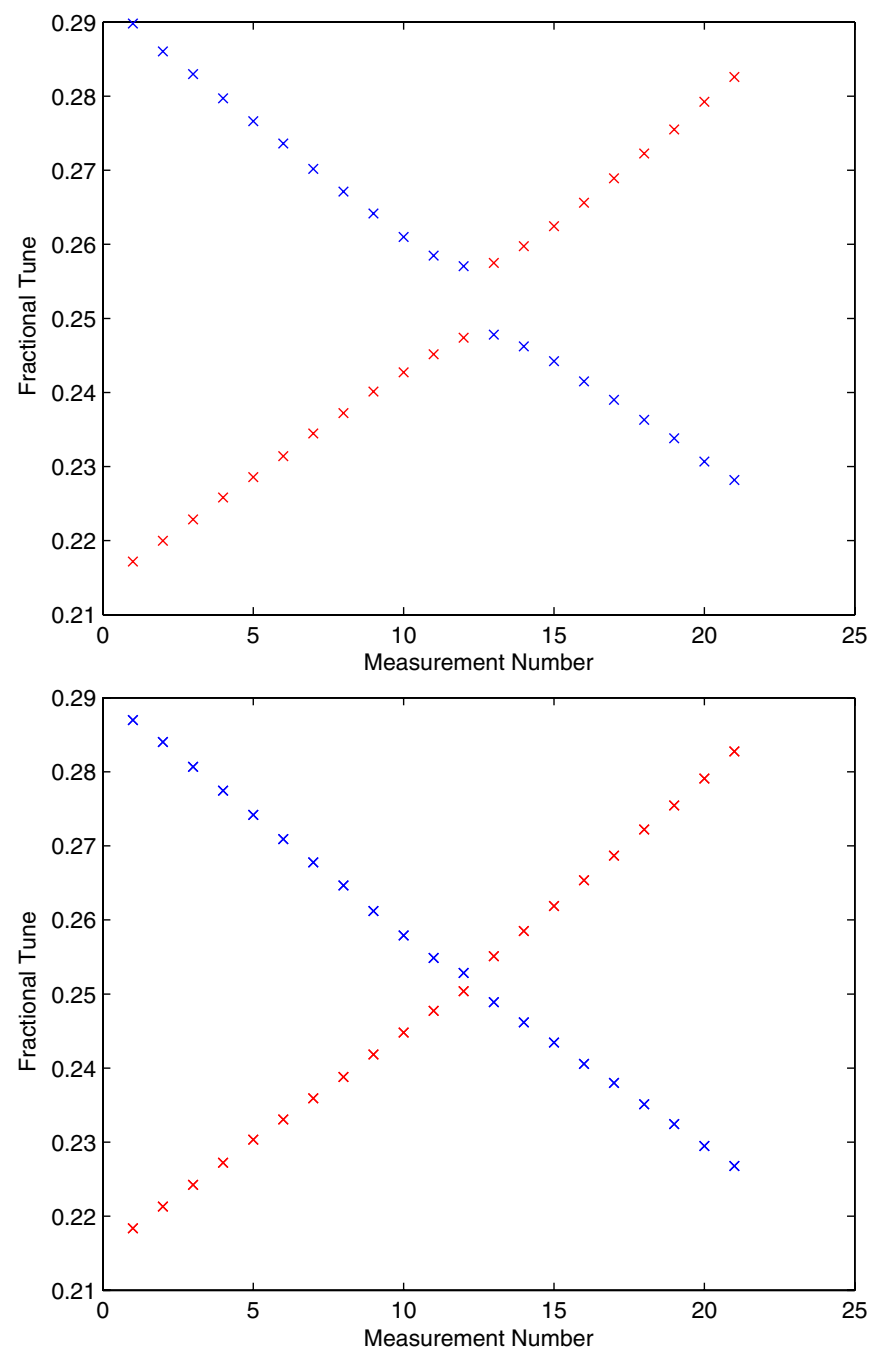

FIG. 7. Tune measurements of horizontal (blue) and vertical (red) tunes as they are brought to resonance. The upper plot shows the $0.9 \%$ coupling measurement and the lower plot shows the $0.07 \%$ measurement.

coefficient and manifests as the tune separation at the difference resonance.

The tunes are measured as they are brought towards a difference resonance at 0.25 and the minimal separation of the tunes is recorded. The tunes are measured by a Fourier analysis of the BPM turn-by-turn data after an excitation from a injection kicker. Some examples of the measurements for different coupling configurations are shown in Fig. 7. During the course of these measurements, it was noticed that the result obtained depended on the chromaticity of the beam. The nominal storage ring lattice settings at the Australian Synchrotron include a high vertical chromaticity to stabilize the beam against resistive wall driven instabilities. It was found that the large tune spread seemed to be allowing the main tune peaks to approach much closer than at lower chromaticities, giving a misleadingly small betatron coupling result. As a result,
TABLE III. Results of tune crossing measurement.

\begin{tabular}{lcc}
\hline \hline $\begin{array}{l}\text { Model } \\
\text { calculated }\end{array}$ & $\begin{array}{c}\text { Measured and } \\
\text { betatron }|C|\end{array}$ & $\begin{array}{c}\text { Betatron } \\
\text { coupling, } g\end{array}$ \\
\hline $0.905 \%$ & $0.0097 \pm 0.0002$ & $0.852 \pm 0.026 \%$ \\
$0.748 \%$ & $0.0091 \pm 0.0002$ & $0.750 \pm 0.021 \%$ \\
$0.406 \%$ & $0.0066 \pm 0.0002$ & $0.396 \pm 0.018 \%$ \\
$0.223 \%$ & $0.0046 \pm 0.0002$ & $0.193 \pm 0.013 \%$ \\
$0.125 \%$ & $0.0034 \pm 0.0002$ & $0.105 \pm 0.010 \%$ \\
$0.074 \%$ & $0.0024 \pm 0.0002$ & $0.053 \pm 0.007 \%$ \\
$0.011 \%$ & $0.0008 \pm 0.0002$ & $0.006 \pm 0.003 \%$ \\
\hline \hline
\end{tabular}

these measurements were taken at low chromaticity $\left(\xi_{x}=\right.$ $\left.\xi_{y}=1.5\right)$ with lower stored current to avoid instabilities. Lowering the chromaticity changes the sextupole strengths which in turn change the skew components from the sextupoles. Consequentially, the coupling settings for the tune crossing measurements are not the same as discussed elsewhere, but are still a valid check of the calibrated model predictions.

The results of measurements of the minimum crossing at several different coupling settings are shown in Table III. The error in the measurement of $|C|$ is dominated by the resolution of the Fourier transform of the BPM data. If we add the effect of vertical dispersion in each of these coupling configurations, we can compare the estimates of the vertical emittance contribution from the betatron coupling and vertical dispersion against the calibrated model prediction. This comparison is shown in Table IV and shows a very good agreement between the calibrated model prediction and the emittance obtained from the measurements. The lowest coupling measurement gives an estimated vertical emittance of $1.32 \pm 0.3 \mathrm{pm}$ and is consistent with the value obtained from the beam lifetime analysis in Sec. VIII.

\section{BEAM TILT MEASUREMENTS}

While the x-ray diagnostic beam line cannot be used to accurately measure the vertical emittance, it does allow us to measure the local beam tilt angle. When we perform a beam envelope calculation on the calibrated model to determine the emittance, the calculation also predicts the local tilt angle of the beam around the ring. If the model is accurately simulating the couplings present, then the predicted and measured local beam tilt should be in very close agreement. To obtain the beam tilt measurements, a series of ten snapshots of the image from the x-ray diagnostic beam line for each of the coupling settings was taken. A two-dimensional Gaussian fit was performed to each image to extract the tilt angle of the beam and an average taken. An example of the images used is shown in Fig. 8. These results were compared with the predicted beam tilt angles derived from the calibrated models. Good agreement between these two data sets will indicate that the calibrated 
TABLE IV. Comparison of calibrated model predictions with vertical dispersion and betatron coupling measurements.

\begin{tabular}{lcccc}
\hline \hline Model $\epsilon_{y}(\mathrm{pm})$ & $\eta_{y}$ component $(\mathrm{pm})$ & Betatron coupling component $(\mathrm{pm})$ & Total measured $(\mathrm{pm})$ & Model measured $(\mathrm{pm})$ \\
\hline $92.31 \pm 2.77$ & $2.24 \pm 0.09$ & $86.90 \pm 2.65$ & $89.55 \pm 2.65$ & $2.76 \pm 3.83$ \\
$76.30 \pm 2.23$ & $2.96 \pm 0.12$ & $76.50 \pm 2.14$ & $78.64 \pm 2.14$ & $2.34 \pm 3.09$ \\
$41.41 \pm 1.24$ & $1.73 \pm 0.07$ & $40.39 \pm 1.84$ & $42.23 \pm 1.84$ & $0.82 \pm 2.22$ \\
$22.75 \pm 0.68$ & $2.04 \pm 0.08$ & $19.69 \pm 1.33$ & $21.72 \pm 1.33$ & $1.03 \pm 1.49$ \\
$12.75 \pm 0.38$ & $2.14 \pm 0.09$ & $10.71 \pm 1.02$ & $12.85 \pm 1.02$ & $0.10 \pm 1.09$ \\
$7.55 \pm 0.23$ & $1.73 \pm 0.07$ & $5.41 \pm 0.71$ & $7.14 \pm 0.71$ & $0.41 \pm 0.75$ \\
$1.05 \pm 0.03$ & $0.73 \pm 0.09$ & $0.59 \pm 0.30$ & $1.32 \pm 0.30$ & $0.27 \pm 0.30$ \\
\hline \hline
\end{tabular}

models, and hence the emittance derived from them, are accurate representations of the real machine.

It should be noted that the measured tilt angle is the angle that the beam image makes with respect to the CCD camera pixels, which have not been calibrated to the axis of the storage ring. Therefore we expect to see a systematic offset between the measurements and predictions. To compensate for this, we found the mean difference between the model and measured values for the data set and subtracted this ( -1.06 degrees) from all data points. It is believed that this subtraction should not hide systematic errors in the model, as they are likely to scale with the coupling and so would show up as a gradient error. To estimate the calibrated model error, the difference in angle due to a source point uncertainty of $10 \mathrm{~mm}$ was calculated. The measurement error is determined by the standard deviation of the fits to the ten images. The results, in Fig. 9, show a very strong agreement between the model predictions and the measurements. A small divergence in the results at each end of the plot indicates there is possibly some systematic error in the model predictions; however, it looks to be a small effect.
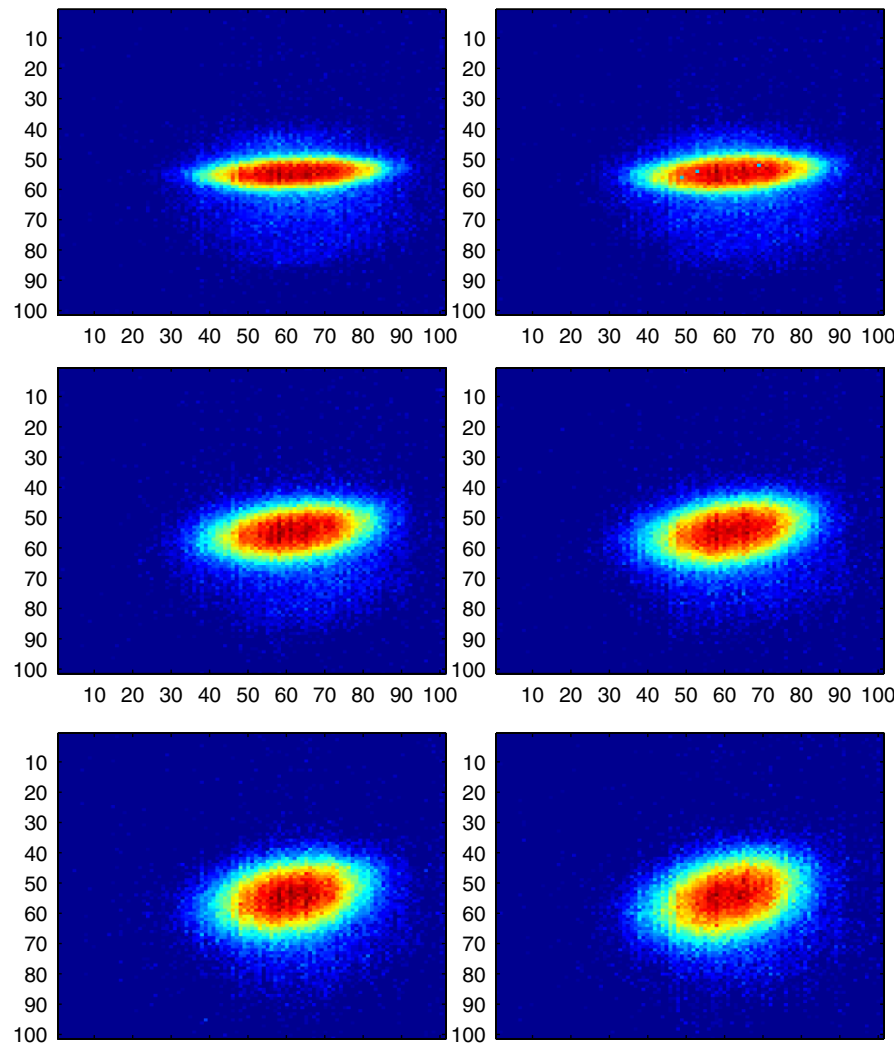

$\begin{array}{llllllllll}10 & 20 & 30 & 40 & 50 & 60 & 70 & 80 & 90 & 100\end{array}$
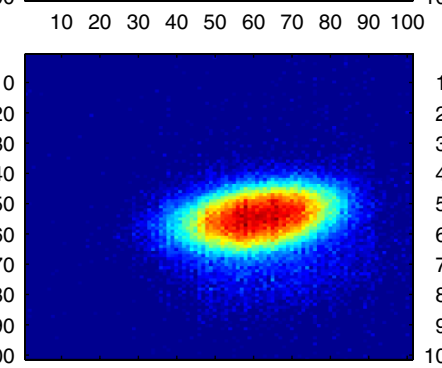

$\begin{array}{llllllllll}10 & 20 & 30 & 40 & 50 & 60 & 70 & 80 & 90 & 100\end{array}$

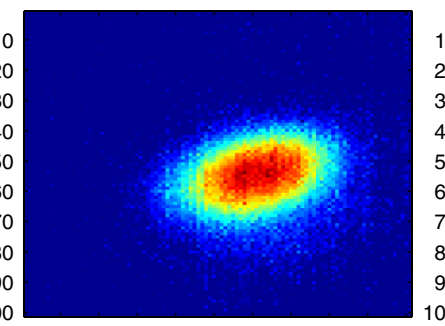

$\begin{array}{llllllllll}10 & 20 & 30 & 40 & 50 & 60 & 70 & 80 & 90 & 100\end{array}$

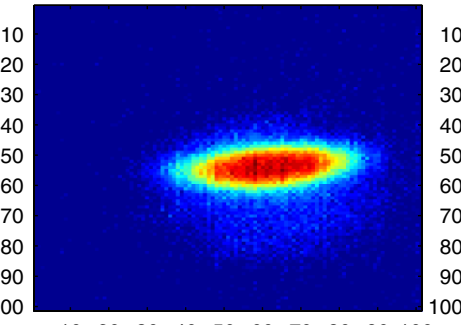

$\begin{array}{llllllllll}10 & 20 & 30 & 40 & 50 & 60 & 70 & 80 & 90 & 100\end{array}$

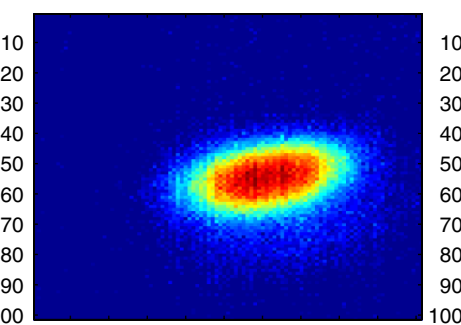

$\begin{array}{llllllllll}10 & 20 & 30 & 40 & 50 & 60 & 70 & 80 & 90 & 100\end{array}$



$\begin{array}{llllllllll}10 & 20 & 30 & 40 & 50 & 60 & 70 & 80 & 90 & 100\end{array}$

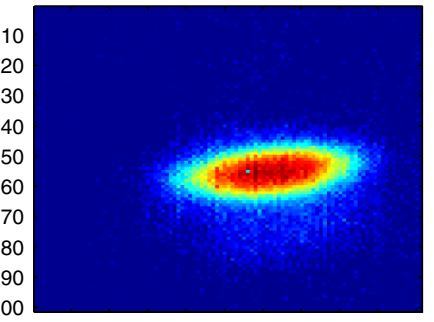

$102030 \quad 4050 \quad 6070 \quad 8090100$

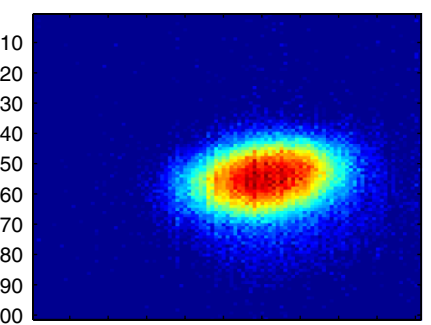

$\begin{array}{llllllllll}10 & 20 & 30 & 40 & 50 & 60 & 70 & 80 & 90 & 100\end{array}$

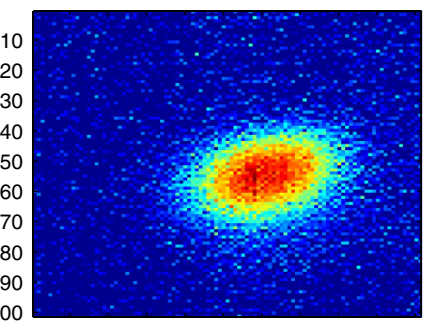

$\begin{array}{llllllllll}10 & 20 & 30 & 40 & 50 & 60 & 70 & 80 & 90 & 100\end{array}$

FIG. 8. Comparison of XDB images for each coupling setting. Minimal coupling is the top left image, and $1 \%$ coupling is in the bottom right. The coupling increases left to right, top to bottom. 


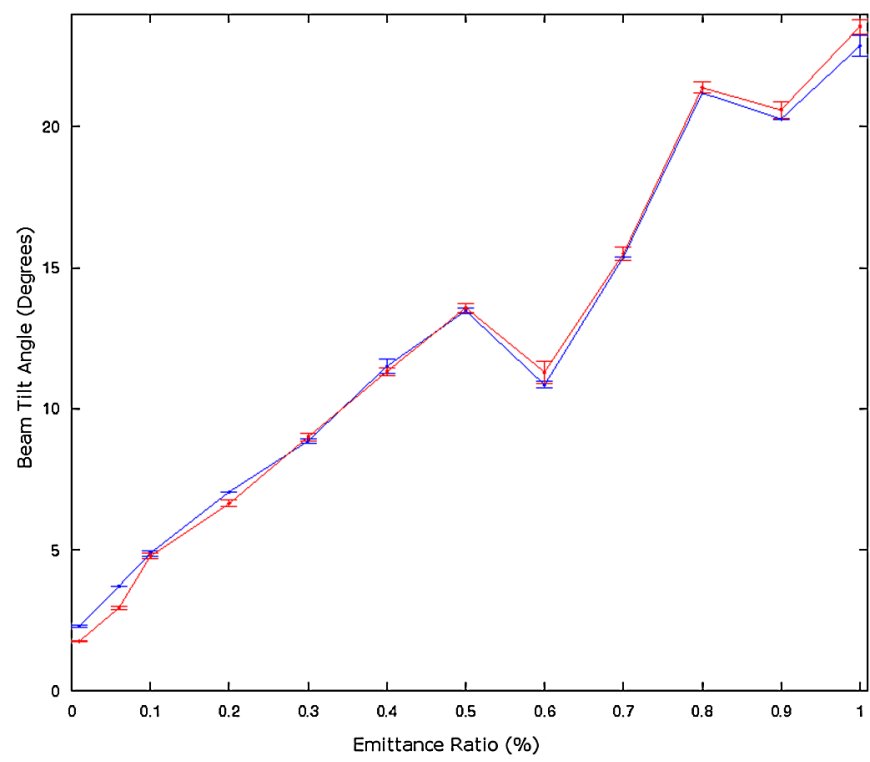

FIG. 9. Measured (blue) and model predicted (red) beam tilt angles at XDB source point. An offset of 1.06 degrees has been subtracted from the measurements to account for the relative tilt of the CCD.

\section{TOUSCHEK LIFETIME MEASUREMENTS}

One beam parameter that is quite straightforward to measure accurately is the beam current and hence lifetime. Since the Touschek scattering cross section depends on the bunch electron density, changes in the volume of the bunch (such as a reduction in the vertical emittance) will directly affect the Touschek lifetime. If we measure the Touschek lifetime of the beam, we should be able to compare it to the theoretical lifetime and deduce an emittance coupling. The Touschek lifetime is given by

$$
\begin{aligned}
\frac{1}{\tau}= & \frac{N r_{e}^{2} c}{8 \pi \sigma_{z} \gamma^{2}}\left\langle\frac{D(\epsilon)}{\delta_{\max }^{3} \sigma_{x} \sigma_{y}}\right\rangle \epsilon=\left(\frac{\delta_{\max } \beta_{x}}{\gamma \sigma_{x}}\right)^{2} \\
D(\epsilon)= & \sqrt{\epsilon}\left(-\frac{3}{2} e^{-\epsilon}+\frac{\epsilon}{2} \int_{\epsilon}^{\infty} \frac{e^{-u} \ln u}{u} d u\right. \\
& \left.+\frac{1}{2}(3 \epsilon-\epsilon \ln \epsilon+2) \int_{\epsilon}^{\infty} \frac{e^{-u}}{u} d u\right) .
\end{aligned}
$$

$N$ is the number of electrons, $r_{e}$ is the classical electron radius, $\sigma_{x, y, z}$ are the rms bunch sizes, and $\delta_{\max }$ is the energy acceptance of the ring as determined by rf acceptance and dynamic aperture. For our storage ring the rf energy acceptance at $3 \mathrm{MV}$ is $2.2 \%$. The dynamic aperture of our lattice is designed to also be $2.2 \%$. A more direct measurement was done by scaling the energy of the storage ring and the measured dynamic aperture was found to be $2.1 \%$. The beam current is measured by a DC current transformer (DCCT) in the storage ring. In order to measure the Touschek component of the lifetime cleanly, we want the beam lifetime to be dominated by Touschek scattering. This is achieved by using a large current
TABLE V. Measurements of Touschek lifetime for different coupling settings.

\begin{tabular}{lc}
\hline \hline LOCO measured coupling & $7 \mathrm{~mA}$ single bunch $\tau(\mathrm{h})$ \\
\hline $0.01 \%$ & $1.49 \pm 0.06$ \\
$0.12 \%$ & $3.15 \pm 0.25$ \\
$0.23 \%$ & $4.13 \pm 0.25$ \\
$0.33 \%$ & $5.58 \pm 0.44$ \\
$0.43 \%$ & $6.35 \pm 0.40$ \\
$0.54 \%$ & $6.76 \pm 0.42$ \\
$0.64 \%$ & $7.29 \pm 0.49$ \\
$0.74 \%$ & $8.14 \pm 0.74$ \\
$0.84 \%$ & $8.55 \pm 0.60$ \\
$0.92 \%$ & $9.01 \pm 0.39$ \\
$1.04 \%$ & $9.16 \pm 0.50$ \\
\hline \hline
\end{tabular}

( $>7 \mathrm{~mA}$ ) single bunch for all of the lifetime measurements.

\section{A. Touschek lifetime vs coupling setting}

As seen from Eq. (2), there is a linear dependence of the Touschek lifetime on the vertical beam size, $\sigma_{y}$. Since $\sigma_{y}=\sqrt{\epsilon_{y} \beta_{y}}$ for negligible vertical dispersion, and $\epsilon_{y} \approx$ $\kappa \epsilon_{x}$ if we vary the ring emittance coupling, $\kappa$, we will change the Touschek lifetime.

Measurements of the lifetime of a single $7 \mathrm{~mA}$ bunch were taken at each of the coupling settings and are shown in Table V. Figure 10 shows a plot of the Touschek dominated lifetime results plotted against the square root of the LOCO determined emittance coupling. A line of best fit has been added and shows a clear linear relationship between the two variables, indicating that the emittance is indeed changing as expected.

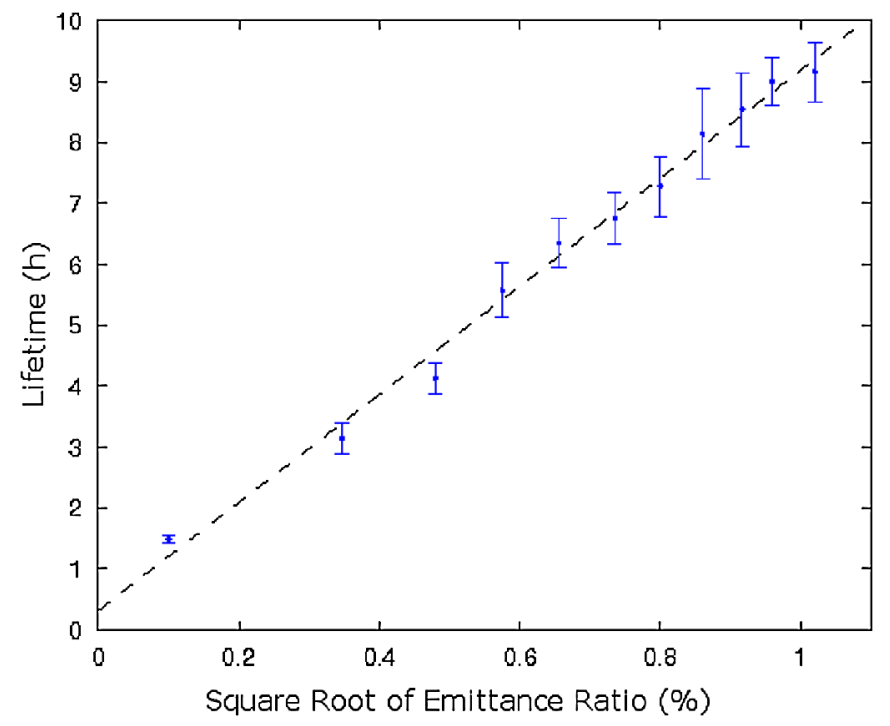

FIG. 10. Plot of Touschek lifetime vs $\sqrt{\kappa}$. Line of best fit shows a clear linear relation. The figure was taken from [14]. 


\section{B. Touschek Lifetime vs rf voltage}

It can also be seen from Eq. (2) that there is a strong dependence on the energy acceptance, $\delta_{\max }$. We can reduce $\delta_{\max }$ by lowering the rf voltage in the storage ring cavities and therefore manipulate the Touschek lifetime. This will give us a series of Touschek lifetime measurements at the same coupling setting. We can then extract the emittance from this data by varying the emittance ratio until the theoretical Touschek parameter curve fits the data.

For this analysis, we filled a single bunch with 8-9 mA of current so that the lifetime was strongly Touschek dominated for the duration of the measurement. We took lifetime data for $10 \mathrm{~min}$, recording the current from the DCCT each second. This was repeated at rf voltages ranging from 3 to $2 \mathrm{MV}$. The actual voltage of the rf at each data point was verified by measuring the synchrotron tune.

The Touschek lifetime parameter was extracted by fitting a decay curve to the DCCT data. The beam current decay fit needs to incorporate the Touschek lifetime's dependence on bunch charge, thus

$$
\begin{gathered}
\frac{d i}{d t}=-\frac{i}{\tau}-\frac{i^{2}}{T} \\
i(t)=\frac{i_{0} T e^{-(t / \tau)}}{T+i_{0} \tau\left(1-e^{-(t / \tau)}\right)},
\end{gathered}
$$

where $T$ is the Touschek parameter $(\mathrm{mAh})$ and $\tau$ is the normal gas scattering lifetime (h). Equation (4) is fit to the DCCT data with a minimization algorithm which varies $T$ and $\tau$ to obtain the best fit via a least squares method. An example of this fit is shown in Fig. 11.

Data were collected for three different coupling settings, the minimum coupling $(0.01 \%)$, uncorrected coupling

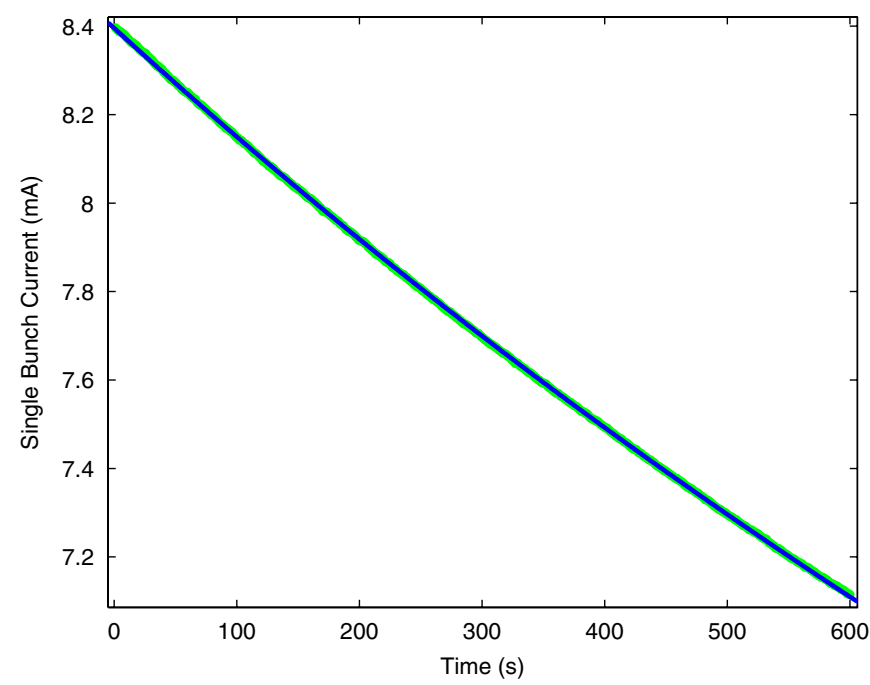

FIG. 11. Example of the beam current decay fit to extract the Touschek component. Data is shown in green; the fit is in blue.

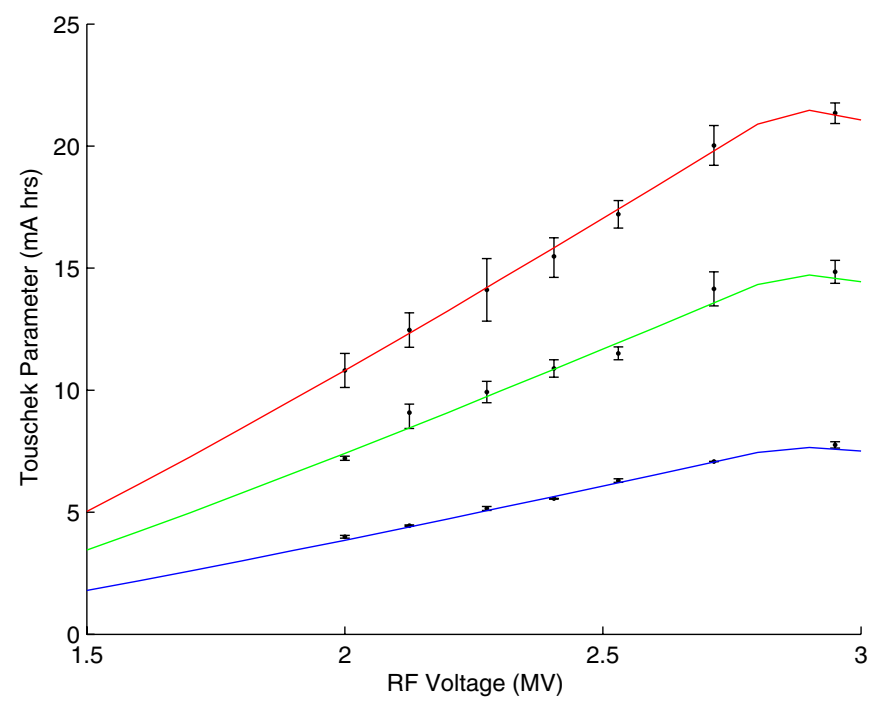

FIG. 12. Measured Touschek parameter at different rf voltages for three coupling settings, $0.1 \%$, uncorrected $(0.06 \%)$ and minimal $(0.01 \%)$. Colored lines are best fit coupling values based on the Touschek equation. Error bars indicate uncertainty in the Touschek parameter from the decay curve fits. Fitted emittance ratio values are $0.0916 \%$ (red), $0.043 \%$ (green), and $0.0116 \%$ (blue).

$(0.06 \%)$, and $0.1 \%$ coupling. The Touschek parameter at each rf voltage was extracted and plotted in Fig. 12. The lines fitted to the data points are the calculated Touschek parameters, using the Touschek equation, with all beam variables in the equation given known values from our lattice. For $\sigma_{z}$, the amount of bunch lengthening due to the high single bunch current was determined by using results from previous single bunch studies [13] at the same current. The only free parameter used to fit the lines to the data points was the emittance coupling ratio, $\epsilon_{y} / \epsilon_{x}$. The results of these fits are summarized in Table VI.

An estimate of the error on the fits was obtained by varying the other beam size parameters, $\epsilon_{x}$ and $\sigma_{z}$ by $\pm 10 \%$. The change in the best fit values for the emittance was determined by refitting the data points and these errors were then added in quadrature. While we believe these parameters are known to a better accuracy, $10 \%$ error was chosen to give a conservative estimate of the total error.

The results from this analysis indicate once again a good agreement between the LOCO calibrated model's estimated vertical emittance and the fitted values. In this

TABLE VI. Results of fits to Touschek parameter data.

\begin{tabular}{lccc}
\hline \hline Set $\epsilon_{y} / \epsilon_{x}(\%)$ & Fitted $\epsilon_{y} / \epsilon_{x}(\%)$ & Fitted $\epsilon_{y}(\mathrm{pm})$ & Fit $\chi^{2} /$ dof \\
\hline 0.01 & $0.012_{-0.002}^{+0.003}$ & $1.2_{-0.2}^{+0.3}$ & 0.69 \\
0.06 & $0.043_{-0.008}^{+0.001}$ & $4.5_{-0.8}^{+1.3}$ & 0.42 \\
0.10 & $0.092_{-0.012}^{+0.025}$ & $9.4_{-1.2}^{+2.6}$ & 0.01 \\
\hline \hline
\end{tabular}


lifetime analysis, the minimum coupling setting has been found to correspond to a vertical emittance of $1.2 \mathrm{pm}$, which is an exceptionally small result, but consistent with the tune crossing measurements and model predictions.

\section{CONCLUSIONS}

It is clear that the key to achieving these measurements lies with good machine alignment, accurate BPMs for orbit measurement, and regular beam based alignment. LOCO has proven to be a very valuable tool in analyzing and optimizing the storage ring lattice. A key step in this analysis was the development of a method that allows setting the emittance coupling to arbitrary values. Having a range of emittance coupling settings to take various measurements has allowed much more in-depth analysis with the indirect methods used.

Both the Touschek analysis and tune crossing measurements indicate the vertical emittance is $1.2-1.3 \mathrm{pm}$, and support the calibrated model's prediction of $1 \mathrm{pm}$. While it is acknowledged that it is difficult to assign a completely reliable number to the vertical emittance from indirect measurements such as these, we can also assign upper limits at a $90 \%$ confidence level based on the estimated errors. The Touschek analysis gives an upper limit of $1.7 \mathrm{pm}$ and the tune crossing measurement gives $1.8 \mathrm{pm}$. These upper limits are currently the smallest vertical emittance reported in a storage ring.

Currently efforts are underway to further improve the alignment of the storage ring magnets, taking into account information from the LOCO based analysis. We hope this realignment will allow us to further reduce the coupling.
We are also investigating possibilities for a more direct emittance measurement.

[1] J. Bolderman and D. Einfeld, Nucl. Instrum. Methods Phys. Res., Sect. A 521, 306 (2004).

[2] M. J. Boland et al., in Proceedings of the 10th European Particle Accelerator Conference, Edinburgh, Scotland, 2006 (EPS-AG, Edinburgh, Scotland, 2006).

[3] M. J. Boland and M. Spencer, in Proceedings of the 11th European Particle Accelerator Conference, Genoa, 2008 (EPS-AG, Genoa, Italy, 2008).

[4] J. McKinlay and B. Barg, in MEDSI2006, 2006, Himeji, Japan, http://medsi2006.spring8.or.jp.

[5] A. Kosicek, in Proceedings of the 21st Particle Accelerator Conference, Knoxville, 2005 (IEEE, Piscataway, NJ, 2005).

[6] J. Safranek, Nucl. Instrum. Methods 388, 27 (1997).

[7] M. Spencer et al., in Proceedings of the 2007 Particle Accelerator Conference, Albuquerque, New Mexico (IEEE, Albuquerque, New Mexico, 2007).

[8] K. Levenberg, Q. Appl. Math. 2, 164 (1944).

[9] Y-R.E. Tan et al., in Proceedings of the 2007 Particle Accelerator Conference, Albuquerque, New Mexico (Ref. [7]).

[10] M. Spencer et al., in Proceedings of the 11th European Particle Accelerator Conference, Genoa, 2008 (Ref. [3]).

[11] K Ohmi et al., Phys. Rev. E 49, 751 (1994).

[12] G. Guignard, Phys. Rev. E 51, 6104 (1995).

[13] R. Dowd et al., in Proceedings of the 11th European Particle Accelerator Conference, Genoa, 2008 (Ref. [3]).

[14] R. Dowd et al., in Proceedings of the 23rd Particle Accelerator Conference, Vancouver, Canada, 2009 (IEEE, Piscataway, NJ, 2009). 